Revue d'histoire de l'Amérique française

REYUE D.HISTOIRE DE L'AMÉRIQUE FRANÇAISE

\title{
La vie à Québec au milieu du XVII ${ }^{\mathbf{e}}$ siècle Étude des sources
}

\section{Jacques Mathieu}

Volume 23, numéro 3, décembre 1969

URI : https://id.erudit.org/iderudit/302907ar

DOI : https://doi.org/10.7202/302907ar

Aller au sommaire du numéro

Éditeur(s)

Institut d'histoire de l'Amérique française

ISSN

0035-2357 (imprimé)

1492-1383 (numérique)

Découvrir la revue

Citer cet article

Mathieu, J. (1969). La vie à Québec au milieu du XVII ${ }^{\mathrm{e}}$ siècle : étude des sources. Revue d'histoire de l'Amérique française, 23(3), 404-424.

https://doi.org/10.7202/302907ar d'utilisation que vous pouvez consulter en ligne.

https://apropos.erudit.org/fr/usagers/politique-dutilisation/ 


\section{LA VIE Å QUÉBEC AU MILIEU DU XVII SIĖCLE ÉTUDE DES SOURCES ${ }^{1}$}

Aucun historien sérieux ne conteste aujourd'hui la nécessité de retourner aux sources originales et de faire la critique des documents consultés pour écrire une histoire véritable, scientifique. Préconisée par les instituts d'histoire de Laval et de Montréal depuis leur fondation, cette méthode scientifique fait maintenant partie du "bagage de connaissances" de la nouvelle génération de chercheurs. De plus, le jeune historien ne se contente plus de raconter; il veut comprendre et expliquer. Pour cela, il fait appel aux sciences humaines connexes à l'histoire; il adopte leurs principes, utilise leurs méthodes et leurs techniques. Il a surtout recours à la sociologie, à l'économique et même à la psychanalyse; un peu, par réaction à la traditionnelle approche politique, événementielle; souvent, pour fournir de nouvelles données et des points de repère en vue de recherches plus poussées; toujours, pour en venir à présenter une image plus complète et plus précise du passé. Voilà certes un résultat enviable !

\section{I - ARCHIVES ET HistoIRE}

Mais n'a-t-on rien oublié au cours de cette évolution ? Si l'on fait consciencieusement la critique interne des documents, il n'en a pas été ainsi de la critique externe. On a tout au plus mentionné que la correspondance officielle des administrateurs canadiens altérait parfois la vérité et dissimulait une partie des faits. On a très rarement cherché à dépasser le document consulté, à bien connaître le cadre (ou le fonds d'archives) dans lequel il s'inscrivait. On s'est attardé à la critique du document comme tel, sans s'élever à la critique de l'ensemble des sources ou des fonds eux-mêmes. C'est pourtant une approche primordiale car sans elle, on ne peut espérer en arriver à une synthèse globale véridique.

Cette critique externe des sources, et non plus seulement d'un document, s'applique d'ailleurs, avec quelques variantes, à toutes les périodes chronologiques du passé, et de plus en plus, à mesure

1 Nous désirons remercier M. Jean Hamelin qui a guidé cette recherche. Son aide fut particulièrement importante pour la précision des notions d'agriculture de subsistance, ou de marché et d'exploitation commercialisée de la terre.

Vol. XxIII, no 3 (décembre 1969) 
qu'on approche du présent. En effet, les documents qui constituent nos archives ont déjà été triés, choisis. L'historien doit donc tenir compte du passage d'un intermédiaire qui a fait une sélection dans les documents et des critères qui ont présidé à ce choix; de la même façon qu'il tient compte de l'orientation d'un journal et de l'opinion du journaliste dans l'étude d'une nouvelle ou d'un éditorial. A la rigueur, il faudrait même aller plus loin. Faut-il rappeler comment la presse anglaise s'est fait prendre par suite d'une erreur de traduction des propos du premier ministre du Canada ? Ou encore le fait que l'audition de l'enregistrement de la dernière conférence fédérale provinciale à laquelle ait participé M. Johnson, a prouvé que par une intonation particulière, le premier ministre du Québec voulait dire exactement le contraire de ce qui a été transcrit. Rappelons enfin que, dans certains cas, il importera même de préciser si un document constitue une entente ou n'est qu'une lettre d'intention.

Il n'est pas question de donner ici une méthode de critique externe des sources, mais il importe d'en préciser quelques aspects particulièrement importants pour l'historien du régime français. Le chercheur ne doit pas se limiter au document qu'il a sous la main. Il doit connaître les documents qui ont été produits et ceux qui ne l'ont pas été. Il est essentiel aussi de savoir quels papiers ont été perdus et pourquoi ils manquent; déterminer si leur absence relève du fonctionnement même de l'administration ${ }^{2}$, d'une carence administrative ${ }^{3}$, de pertes dues aux avatars de l'histoire ${ }^{4}$ ou d'une mauvaise conservation tout simplement.

Seule, la connaissance approfondie du fonctionnement de l'administration permettra alors de replacer le document dont le chercheur dispose, dans un cadre plus vaste. L'histoire globale est à ce prix: une période de recherche intensive dans l'ensemble des documents produits par une administration, un contrôle des avancés par l'étude des fonds d'archives eux-mêmes. Car, un document est créé par et pour une administration; il reflète l'activité et le fonctionnement de cette administration, sa compétence et sa juridiction. ${ }^{5}$

${ }^{2}$ C'est le cas des exploits d'assignations, des requêtes à l'Intendant et des rapports d'enquête des conseillers du Conseil souverain.

3 Ainsi, on ne tenait pas de registres de port, d'ailleurs inutiles à l'administration courante: les difficultés avec l'équipage ou les commerçants et les cas d'avaries devant être déclarés et enregistrés par la cour. Les registres portuaires n'avaient donc pas une valeur permanente comme les titres de propriétés. de documents.

${ }^{4}$ Par exemple: les feux ou le changement d'allégeance et le transfert

5 C'est pourquoi d'ailleurs la classification des Archives reconstitue les structures et les compétences administratives. 
Cette vérification, essentielle à toute étude, devient primordiale à toute tentative de synthèse globale pour éviter de présenter une image faussée de la Nouvelle-France, par suite de généralisations faites à partir d'une seule série de documents. La relation quasi automatique établie entre les ordonnances des intendants et les lois d'un gouvernement moderne illustre bien ce type d'erreurs. L'analyse des ordonnances permet de constater la présence d'un grand nombre d'actes se rapportant à la terre, aux limites des seigneuries, aux droits et devoirs des seigneurs et des censitaires. Il semble logique alors de conclure à l'importance de la culture de la terre au Canada sous le régime français et même, à l'existence d'une société agraire. Mais replaçons ces ordonnances dans le cadre de l'administration gouvernementale. On conçoit bien qu'un litige entre des marchands se réglait devant une cour de justice à moins de eas vraiment exceptionnels. De plus, les contraventions aux règlements commerciaux faisaient l'objet d'une poursuite en cour par l'agent de la compagnie qui détenait le monopole et réclamait le respect de ses prérogatives. On avait donc rarement recours à l'intendant. Par contre, le seigneur récalcitrant à remplir ses devoirs avait directement affaire à l'intendant. Or, grâce aux ordonnances, ce dernier disposait d'un moyen beaucoup plus rapide et efficace, peut-être même plus prestigieux que le Conseil souverain pour exprimer ses volontés. S'il reste vrai que les ordonnances des intendants ont souvent rapport à la terre, il devient fort restrictif, sinon faux, de parler de société agraire. Erreur fréquente donc qui s'explique par l'absence de critique externe des sources, la méconnaissance de l'administration et l'absence de certaines séries de documents. ${ }^{6}$

Cet aspect de la critique des sources est d'autant plus important pour l'historien du régime français qu'il peut consulter sur microfilm les fonds d'archives conservés ou transférés en France et bénéficier de sources documentaires qui permettent de suppléer à celles qui ont été perdues et même, en certains cas, de documents qui n'ont pas été produits par l'administration canadienne. ${ }^{7}$ Il faut enfin essayer d'évaluer les carences administratives en tenant compte des besoins de l'administration, des diffi-

${ }^{6}$ En ce sens, la décision de 1760 , qui répondait à des besoins administratifs, de conserver au pays les documents justifiant un statut civil ou un titre de propriété plutôt que les registres à caractère financier a peut-être influencé notre historiographie.

7 C'est le cas des registres de ports français qui permettent de reconstituer le trafic maritime du Canada et qui contiennent des listes de propriétaires et de capitaines de navires en plus du détail des cargaisons, en quantité, qualité et valeur. 
cultés particulières au Canada, de la mentalité de l'époque et des retards dans l'adaptation aux réalités nouvelles - retards qui semblent d'ailleurs chroniques dans les administrations gouvernementales - . Ainsi, Robert Mandrou a signalé comment les théories mercantilistes ont perduré dans la mentalité et l'administration française. ${ }^{8}$ Pour le Canada, on pourrait prendre le modèle et le programme de recensement de Vauban, introduit dès 1685 , mais qui ne sera plus rigoureusement appliqué ${ }^{9}$; ou, encore une fois, la négligence à tenir des registres de port.

Evidemment, cette étude des sources n'est pas une condition sine qua non à la production d'une œuvre de valeur. Certaines monographies sont quasi définitives; elles vaudront en tout cas pendant encore une génération, mais l'histoire globale du régime français reste impossible à faire tant qu'on n'aura pas étudié à fond le fonctionnement des administrations et les documents qu'elles ont produits.

Pratiquement, les historiens de la Nouvelle-France devraient orienter leur recherche vers l'utilisation des sources nouvelles, mises à leur disposition grâce aux microfilms, et vers les séries de documents encore peu utilisées, mais d'une richesse incomparable, comme les registres de cour de justice et les actes notariés. Bien sûr, pour mener une recherche semblable à bonne fin, il faut une équipe, et beaucoup de travail, mais les résultats en valent la peine, car, quelles que soient les approches, on n'aura de bons résultats qu'en connaissant la formation, le but et la portée des documents. Enfin, à mon avis, un bon article, des points de repères précis valent plus pour l'histoire du Canada qu'une œuvre monumentale fautive parce que mal orientée au départ ou basée sur des postulats erronés.

C'est dans cette optique d'histoire globale et de critique externe des sources que je veux étudier La vie à Québec au milieu $d u X V I I^{e}$ siècle. Titre bien vaste, qui recouvre cependant une réalité plus mince: nous nous limitons à deux années (16671668), à un survol de l'ensemble des sources publiques disponibles et des champs d'activité de la population; nous essayons toutefois de quantifier les secteurs de travail et l'occupation des gens plutôt que d'utiliser des témoignages à caractère qualitatif trop souvent partiaux.

${ }^{8}$ Robert Mandrou, La France aux XVII et $X V I I I^{e}$ siècles (Paris, PUF, 1967), 111.

${ }^{\circ}$ Louise Dechêne, La correspondance de Vauban relative au Canada (Québec, Ministère des Affaires culturelles, 1968), 4-5. 


\section{II - LES SOURCES OFFICIELLES : FORMATION, CONTENU, LIMITES}

Les édits et ordonnances du roi ${ }^{10}$ illustrent l'état de tutelle de la Nouvelle-France par rapport à la métropole. On y trouve aussi bien des documents relatifs à des problèmes immédiats qu'à des politiques générales. Dans l'un et l'autre cas toutefois, certains arrêts n'ont aucunement influencé l'administration ou la vie au Canada, et même ne correspondaient pas toujours à la réalité. Ainsi en est-il des Edits de Marly en 1711 faussement basés sur les pouvoirs des seigneurs et qui plus est, ne furent pas respectés, ou de la dépendance, qui s'est avérée toute théorique, des commissaires ordonnateurs de l'Ile Royale et de la Louisiane envers l'intendant de la Nouvelle-France. Encore plus probants sont les édits royaux qui supprimèrent, puis rétablirent officiellement la Prévôté de Québec en 1674 et 1677 alors que cette cour de justice fut plus active que jamais au cours de cette période de suspension, ${ }^{11}$ sans compter le refus d'insinuer et donc d'appliquer certains édits. ${ }^{12}$

\section{Les ordonnances des intendants et des gouverneurs ${ }^{13}$}

Il n'est pas certain que nous possédions toutes les ordonnances émises par le "gouvernement" de la Nouvelle-France en 1667 et 1668, car elles ne furent pas transcrites dans des registres officiels avant 1705. De plus, l'imprécision des tâches se retrouve constamment à ce niveau. Les ordonnances furent le fait de Talon, de Tracy, ou du triumvirat Tracy, Courcelles et Talon; elles avaient une portée plus ou moins grande et on en trouve, en outre, à d'autres niveaux de l'administration. Ce dernier

$10 \mathrm{AQ}, \mathrm{NF}-16,9$ vol. Plusieurs pièces ont été publiées dans: Edits, ordonnances royaux, déclarations et arrêts du conseil d'état du roi concernant le Cianada (Québec, 1854), vol. I. - Arrêts et règlements du Conseil supérieur de Québec et ordonnances et jugements des intendants du Canada. (Québec, 1855), vol. II. - Complément des ordonnances et jugements des gouverneurs et intendants du Canada précédé des commissions des dits gouverneurs et intendants et des différents officiers civils et de justice (Québec, 1856), vol. III.

11 J.-B. Gareau, "La Prévôté de Québec, Ses officiers — ses registres", RAPQ (1943-44): 56 .

12 Cameron Nish, La bourgeoisie canadienne 1729-1748. Caractères et fonctions. Thèse manuscrite, s.l., s.d. (Université Laval, 1968), 164 et 200.

$13 \mathrm{AQ}, \mathrm{NF} 1$ et 2. En partie publié dans: Pierre-Georges Roy, Ordonnances, commissions, etc., etc., des gouverneurs et intendants de la NouvelleFrance, 1639-1706 (Beauceville, L'Eclaireur, 1924), 2 vol. - Pierre-Georges Roy, Inventaire des ordonnances des Intendants de la Nouvelle-France conservées aux Archives provinciales de Québec (1706-1760) (Beauceville, L'Eclaireur, 1919), 4 vol. - Pour la période étudiée, 1667-1668, les ordonnances sont publiées dans le premier volume: 51-76. 
facteur est d'ailleurs capital pour préciser les préoccupations des administrateurs de la colonie.

\section{La correspondance générale ${ }^{14}$}

Les lettres et les mémoires de Talon (comme ceux de tous les autres intendants et gouverneurs) ne relatent pas tout. Ils pèchent surtout par omission: on ne tenait pas en effet à dévoiler des activités plus ou moins honnêtes. De plus, ces documents ne livrent souvent que des bribes de la vie à Québec. On pensait bien plus à l'avenir qu'à décrire l'activité même des gens. On vivait de projets. Il n'est qu'à considérer le “Mémoire de Talon sur l'état présent du Canada (1667)" ${ }^{15}$ pour s'en persuader. Ayant décrit le Canada en 3 paragraphes, l'administrateur en consacre le double aux possibilités de développement. Par la suite, il accorde $11 \frac{1}{2}$ page à la description de l'état ecclésiastique et surtout à la nécessité de limiter le pouvoir des religieux sur le temporel, mais il règle le cas du peuple en 7 lignes.

\section{Les actes notariés ${ }^{16}$}

Les actes notariés, peu utilisés, souvent décrits à la légère comme les documents les plus intéressants de notre histoire parce qu'aucune autre série de documents n'illustrerait mieux ni plus complètement la vie même du peuple, constituent assurément une mine de renseignements fort utiles, mais ils doivent être soumis à une critique externe rigoureuse. De prime abord, il faut signaler que tout n'était pas objet d'un acte devant notaire, d'où la nécessité d'apporter des nuances majeures à l'étude quantitative de ces données. Nuance que la comparaison avec d'autres sources rend heureusement possible. Ainsi les greffes des notaires exerçant leur profession dans la région de Québec en $1667^{17}$ comprennent moins de 5 engagements sur un total de 313 actes. Le recensement de $1666^{18}$ révèle pourtant la présence à Québec de 441 hommes servant comme domestique, engagé, volontaire

${ }^{14}$ Ces documents constituent les séries $\mathrm{B}$ et $\mathrm{C}^{11 \mathrm{~A}}$. Ils sont conservés en microfilm aux Archives du Québec.

15 "Correspondance échangée entre la cour de France et l'intendant Talon pendant ses deux administrations dans la Nouvelle-France", RAPQ (1930-31) : 63-66.

16 Tous les greffes des notaires ayant exercé leur profession à Québec avant 1800 sont conservés aux Archives du Québec; les autres greffes sont dispersés dans les 35 districts judiciaires.

17 Il s'agit des greffes des notaires Claude Aubert, Romain Becquet, Pierre Duquet et Paul Vachon, dont les répertoires ont été publiés par Pierre-Georges et Antoine Roy dans la série des Inventaires des greffes de notaires du régime français, tomes I et II.

18 Ce recensement a été publié dans le Rapport des Archives de la Province de Québec (1930-31) : 1-154. 
ou travaillant. Il en est également ainsi pour les ententes à caractère commercial qui semblent se faire à l'amiable d'autant plus que les échéances sont à court terme: elles font donc rarement l'objet d'actes notariés. Il faut aussi se méfier des contrats qui n'ont pas de suite. C'est le cas des concessions de terre en série à des gens qui quittèrent Québec assez tôt et ne laissèrent pas d'autres traces de leur séjour dans la colonie. ${ }^{19}$ Signalons tout de suite que bon nombre de concessions de terre peuvent en partie s'expliquer par la mentalité française qui attachait beaucoup d'importance à la terre et y voyait un élément sécuritaire, par la législation, ${ }^{20}$ et surtout par la nécessité de prouver légalement ses titres de propriété.

\section{Le Conseil souverain ${ }^{21}$}

Cour de justice supérieure qui agit comme cour d'appel et joue un rôle important dans l'administration de la colonie, le Conseil souverain donne une image partielle de la société québécoise, car on n'y retrouve qu'un nombre restreint d'individus, surtout des criminels, et les personnages importants de la colonie. Les renseignements précis sur la société sont aussi assez rares car le greffier n'a généralement transcrit au registre que l'arrêt rendu par la cour. On ne dispose donc ni des exploits d'assignations, ni des requêtes présentées sous forme de grief et moyen d'appel; et on déplore surtout l'absence de tous les rapports faits par des "conseillers-enquêteurs". ${ }^{22}$ Enfin il n'y a que

19 Voir à ce sujet l'inventaire publié du greffe de Romain Becquet aux pages 271 à 274. Plusieurs concessionnaires de terre ne figurent ni dans le Recensement de 1666, ni dans le Dictionnaire de Tanguay annoté par Archange Godbout et Roland-J. Auger.

20 Une ordonnance de la Prévôté de Québec en 1667 oblige les volontaires à prendre un maître ou une concession. Registres de la Prévôté de Québec, I: f.93v.

$21 \mathrm{AQ}, \mathrm{NF}-11,13$ et 14 . La première partie a été publiée dans: Province de Québec, Jugements et délibérations du Conseil Souverain de la Nouvelle-France, 1663-1716 (Québec, 1885-1891), 6 vol. - Pierre-Georges Roy a fait un Inventaire des jugements et délibérations du Conseil supérieur de la Nouvelle-France de 1717 à 1760 (Beauceville, 1932-1940), 7 vol. Il existe, de plus, aux Archives du Québec un inventaire manuscrit des pièces détachées du Conseil supérieur.

22 Il arrivait fréquemment que par un arrêt le Conseil souverain "commettait" un conseiller pour étudier un conflit entre deux individus ou pour enquêter sur une possibilité de contravention à la loi. Mais les parties ne revenaient pas toujours en cour: le problème s'étant probablement réglé devant le conseiller ainsi commis. D'ailleurs, à une occasion au moins, un arrêt précise que le conseiller devra entendre et régler définitivement un litige (Province de Québec, Jugements et délibérations du Conseil souverain de la Nouvelle-France, 1663-1716, I: 416). De plus, le procès-verbal inscrit au registre commence parfois par les mots suivants: "Veu les informations faites..." et il ne reste rien de cette enquête. A titre d'exemple, voir: Province de Québec, Ibid.: 449. 
124 procès-verbaux comprenant 15 ordonnances et 25 ordres d'insinuations.

\section{La Prévôté de Québec ${ }^{23}$}

La meilleure source de renseignements sur la vie à Québec au milieu du XVII ${ }^{\mathrm{e}}$ siècle se trouve dans les registres de la Prévôté de Québec. Les registres de cette cour civile et criminelle de première instance sont, pour cette période, plus riches que toute autre source connue, car ils touchent de plus près une plus grande portion de la population: deux fois plus que les actes notariés. ${ }^{24}$ Les registres de la Prévôté de Québec en 1667 et 1668 contiennent exactement 1490 procès-verbaux dont environ 1200 sont des litiges, faisant 800 à 900 causes et concernant plus de 1100 personnes. De plus, les parties en cause sont bien identifiées - on donne souvent l'occupation et le lieu de résidence des gens qui comparaissent - et le greffier enregistre généralement les prétentions des deux parties en plus de la sentence rendue. Les procès-verbaux de ces registres contiennent en outre, des ordonnances et des avis de portée aussi grande que celles émises par les juridictions supérieures: gouverneur, intendant et Conseil souverain.

Un si grand nombre de procès, en deux ans seulement, permet de mieux caractériser la population québécoise ${ }^{25}$ et même d'apporter certaines précisions préalables aux études démographiques.

\section{III - LA VIE À QUÉBEC}

\section{A. Population}

Quelle valeur peut-on accorder au recensement de 1666 ? Nul doute qu'on y trouve des données très utiles à la connaissance de notre passé, mais jusqu'à quel point est-il complet ? La comparaison de la liste des gens figurant au recensement avec l'index tiré des registres de la Prévôté permet de préciser cet inconnu. ${ }^{26}$ Le recensement de 1666 permet de dénombrer 885

${ }^{23} \mathrm{AQ}, \mathrm{NF}-19$ et 20 . Des inventaires de ces documents sont en voie de réalisation.

24 Cette proportion peut toutefois être inversée au XVIIIe siècle.

25 Il me semble que ceux qui font de la sociologie historique ou de l'histoire sociale auraient grand intérêt à utiliser les registres de cour de justice inférieure. Le nombre et le type de procès en rapport avec le chiffre de la population permettraient d'établir avec précision les étapes de l'évolution d'une société. Ainsi, le grand nombre de procès à Québec en 1667 prouve, sans doute possible qu'on ne peut plus parler de colonie-comptoir pour cette époque.

26 Pour plus de sûreté et par manque de temps, il n'a été tenu compte que de la population masculine de plus de 16 ans. 
mâles de plus de 16 ans dans le gouvernement de Québec, soit le nombre maximum d'individus qui logiquement pouvaient se présenter en cour. Or, un peu plus de 900 hommes figurent à l'index des registres de la Prévôté de Québec pour les années 1667 et 1668. Mais tous n'allaient pas devant la justice: c'est le cas d'un bon nombre de jeunes hommes vivant avec leurs parents, de 206 domestiques et engagés, des 72 ecclésiastiques et de leurs hommes de travail; au total, 459 hommes de plus de 16 ans qui figurent dans le recensement de la région de Québec en 1666 ne se présentèrent pas devant la Prévôté au cours des deux années suivantes. Ajoutons ces 459 personnes recensées, absentes des procès-verbaux de la Prévôté, aux 900 qui s'y présentèrent, la population masculine de plus de 16 ans dans la région de Québec atteint alors le chiffre de 1359 , soit $50 \%$ de plus que le nombre recensé. Voilà un chiffre étonnant et fantastique, d'autant plus que les facteurs qui doivent entrer en ligne de compte semblent s'équilibrer.

Facteurs susceptibles d'augmenter ce nombre:

- Des individus peuvent ne figurer ni au recensement ni dans les registres de cour. Ainsi dans le seul greffe de Romain Becquet, et pour la seule année 1667, j'ai relevé 16 concessionnaires de terre qui ne figurent ni dans le recensement, ni dans le Tanguay annoté par Archange Godbout et Roland-J. Auger, ni dans les registres de cour de la Prévôté. En fait les documents de contrôle ne couvrent que deux années (1667-1668) et n'ont été tirés que d'une seule source: les registres de la Prévôté de Québec.

- L'étude des types de cause démontre et suppose presque toujours la nécessité, pour ceux qui comparaissent, d'un séjour suffisamment long à Québec pour avoir contracté des engagements et y avoir fait défaut, ce qui porte à croire que plusieurs étaient à Québec en 1666.

\section{Facteurs susceptibles de diminuer ce nombre:}

- Décès.

- Départs vers la France, vers Trois-Rivières ou Montréal ou pour faire la traite des fourrures.

- L'absence des soldats dans le recensement. Ils semblent toutefois très peu nombreux devant la cour: une vingtaine peut-être.

Il faut aussi tenir compte des Québécois qui firent partie de l'expédition de Courcelles. Ayant quitté Québec le 9 janvier 1666, avec 600 hommes dont 45 Canadiens, dit Gustave Lanctôt, Cour- 
celles n'y revint pas avant la mi-mars. ${ }^{27}$ Or, Cyprien Tanguay "a établi de façon indiscutable... que le dénombrement de 1666 avait été fait dans les mois de février et mars". ${ }^{28}$

Il y avait donc à Québec plus d'hommes qu'on ne l'a dit et cela, sans compter les soldats français. Il me semble alors un peu illusoire de prétendre faire des études démographiques à partir de la seule source que constitue le recensement de 1666.

Une autre conclusion se dégage de cet aperçu de la population: sa grande mobilité. Ces gens qui venaient et repartaient, qui passaient sans laisser beaucoup de traces, n'en influencèrent pas moins la vie québécoise. Liée aux facteurs géographiques d'immensité et de dispersion, cette mobilité rendait utopique et irréalisable toute administration et toute règlementation non basées sur des lois réalistes et simples, pour ne pas dire naturelles. Ainsi, au Conseil Souverain, trois couples demandèrent l'insinuation de leur contrat de mariage après les délais fixés par l'ordonnance, en expliquant qu'ils n'étaient pas au courant de l'existence de ce règlement.

Mais quelle était la composition de cette population ? Talon mentionnait trois états: l'ecclésiastique, le noble et le populaire. D'une part, les ecclésiastiques avaient un champ d'action bien défini, d'autre part il n'y avait que 8 nobles dans toute la Nouvelle-France. Reste le peuple qui constituait la majeure partie de la société et que les historiens ont étudié et classifié. Je ne veux pas reprendre la répartition déjà faite - celle de Marcel Trudel, bien que résumée, me semble à point - L L'étude des sources révèle toutefois des facteurs qu'il importe de souligner. Le gouvernement de Québec comptait plus de domestiques et d'engagés que de ménages. Or, le statut social de ces domestiques était mal défini. D'une part, ils constituaient une catégorie sociale inférieure, d'autre part, ils avaient des droits auxquels ils tenaient fermement. Ainsi, le lieutenant général de la Prévôté de Québec condamna trois femmes accusées d'avoir battu un domestique à faire amende honorable au maître de cet engagé; ${ }^{29}$ jugement que le Conseil souverain renversa au profit du domestique humilié. Par contre, l'un d'entre eux refusa de payer un matelas parce que d'autres y avaient couché. ${ }^{30}$ Enfin, dans

\footnotetext{
${ }^{27}$ W. J. Eccles, "Rémy de Courcelle", Dictionnaire biographique $d u$ Canada (Québec, 1966), I: 583-585.

28 "Le premier recensement de la Nouvelle-France", dans RAPQ (1935-36) : 1-2.

29 Registres de la Prévôté de Québec, 1667, 2e partie, f.29.

30 Ibid., f.27.
} 
ces règlements sur la justice, Talon traita longuement des relations maître-domestique. ${ }^{31}$

Les hommes de métier, par contre, jouissaient d'un statut assez élevé et cherchaient à protéger leurs intérêts particuliers, un peu sur le modèle des corporations de métier en France. Ainsi, quand un charpentier refusa de faire un plancher et affirma qu'aucun homme de métier à Québec ne ferait ce travail, le procureur général intervint vigoureusement pour dénoncer cette "pratique des artisans". ${ }^{32}$ De plus, un homme de métier appelé à témoigner sur la construction d'une cheminée ne manqua pas de signaler que ce type de travail devrait être réservé à des experts. ${ }^{33}$

Le statut légal de la femme était aussi imprécis. Si elle pouvait prendre action en justice et représenter d'autres personnes, un homme pouvait se dégager de tout engagement et de toute dette contractée par sa femme s'il avait pris la précaution d'avertir les marchands de ne conclure aucune transaction avec elle. ${ }^{34}$

Signalons enfin l'absence quasi totale d'Indiens dans les documents administratifs de la Nouvelle-France. Il n'y a que deux mentions dans les registres de cour et aucune dans les actes notariés. Leur absence reflète une imposition de structures aux coloniaux français dont s'exemptèrent les Amérindiens et un refus de la population indigène à s'assimiler ou à se laisser assimiler; elle manifeste surtout l'existence de deux mondes totalement distincts par leur mode de vie, leur aspiration et leur gouvernement.

\section{B. L'ACTIVITÉ DE LA POPULATION}

L'étude des occupations de cette population est encore plus intéressante. Aux deux secteurs d'activité bien connus, la traite des fourrures et l'agriculture, s'ajoutent le commerce et la justice.

Il est inutile d'épiloguer bien longtemps sur le commerce des pelleteries, car, bien que base du développement de la colonie, Québec n'en est touché qu'indirectement après 1650 .

\section{L'agriculture}

La culture de la terre constituait aussi un besoin vital pour la colonie. Mais ce mode de vie occupait-il la majorité de la popu-

31 Pierre-Georges Roy, Ordonnances, commissions... op. cit., 53-54.

32 Registres de la Prévôté de Québec, $1667,1^{\mathrm{e}}$ partie, f.44.

33 Ibid., 1668, f.110v. et $117 \mathrm{v}$.

34 Ibid., $1^{\mathrm{e}}$ partie, f.34v. 
lation québécoise, et en fait, pratiquait-on une agriculture de subsistance ou produisait-on en vue des marchés? Les deux grandes séries de documents qui touchent le peuple de près, soit les actes notariés et les registres de cour de la Prévôté permettent de préciser l'importance et la nature de cette agriculture. ${ }^{35}$

\section{Prévôté de Québec 1667-1668}

Sujet

Nombre

procès-verbaux

- Echange ou vente de marchandises et produits

300

- Réclamations d'argent sans indication de motifs

223

(probablement travail ou vente)

- Travail des hommes de métier

- Dettes

- Jouissance de maison et de terre

- Limites de terre

Actes notariés 1667, Québec

Sujet

Nombre de d'actes

Concessions de terre

85

- Vente ou échange de terre et maison

- Contrats de mariage

- Obligations

24

- Marchés

- Ventes diverses

La comparaison des données tirées de ces deux séries de documents met en opposition directe et frappante l'activité agricole et l'activité commerciale. Un facteur relevant de la critique externe des documents pourrait toutefois modifier la portée de nos conclusions sur l'importance relative de ces secteurs d'activité: l'absence, pour la période étudiée, des registres de cours de justice seigneuriale; lacune d'autant plus grave qu'elle pourrait produire des chiffres inverses à ceux tirés de la Prévôté. ${ }^{36}$

${ }^{35}$ Les tableaux suivants ne comprennent pas les actes ou procèsverbaux peu nombreux et portant sur des sujets divers comme les donations et inventaires de biens, les quittances, les vols, les bagarres et les procès de succession. L'analyse détaillée de ces pièces, bien que fort intéressante, dépasse les cadres de ce travail.

36 Il faut toutefois signaler qu'un chercheur étudiant une période postérieure à celle-ci pourra utiliser les registres de la cour seigneuriale de Notre-Dame-des-Anges qui remontent à 1689 et ceux du bailliage de la Côte-de-Beaupré qui datent de 1683. Les premiers sont conservés aux Archives du Québec; les seconds au Séminaire de Québec. 
Deux caractéristiques de l'occupation de la terre se dégagent toutefois de l'analyse des sources consultées: son aspect sécuritaire et son caractère commercial. Nous avons retracé le curriculum vitæ de 90 de 115 concessionnaires de terre en 1667 et 1668: ${ }^{37}$ la plupart, d'anciens engagés ou de jeunes Canadiens. 43 des 90 concessionnaires connus se sont mariés dans les deux années suivant l'acte de concession et 14, dans les quatre années précédant cette concession. A quelques exceptions près, les autres terres furent acquises par des pères de famille qui avaient un ou des fils entre 12 et 18 ans. Ce lien très étroit entre mariage, établissement des jeunes hommes et concession de terre, illustre assez bien l'élément de sécurité que comportait la possession de la terre.

D'un autre côté, il existe plusieurs preuves que l'habitant canadien cultivait la terre pour sa subsistance, bien sûr, mais disposait d'une surproduction suffisante pour tirer d'intéressants profits du marché local. Dans son mémoire de 1667, Talon mentionnait les possibilités d'échanges de denrées à Québec. ${ }^{38}$ Le grand nombre d'engagés et de domestiques vivant dans les fermes indique aussi cette tendance à produire le plus possible. Pierre Boucher, pour sa part, recommandait aux immigrants moyennement pourvus d'amener deux hommes pour défricher et cultiver. De plus, l'agriculteur canadien bénéficiait, sans concurrence extérieure, d'un marché assuré et assez important: fonctionnaires du gouvernement, coureurs de bois, hommes de métier vivant de leurs services, membres d'équipages des navires et même, à l'occasion, les troupes françaises. ${ }^{39}$ Enfin, malgré les apparences, l'analyse des causes entendues par la Prévôté de Québec ne dément pas le caractère commercial de l'agriculture en NouvelleFrance; au contraire, elle le fortifie. En effet, nombre de réclamations sur l'échange ou la vente de marchandises et de produits concernent la fourniture de produits de consommation comme l'anguille, la viande, le blé, les pois et le tabac, sans compter les articles achetés par les habitants comme bestiaux, haches, habits, souliers, raquettes, meubles, canots, sel et eau de vie. Il y a en plus une trentaine de procès relatifs à la location d'hommes, d'animaux et de services pour les besoins de la ferme. Obtenir de bons rendements du sol était donc une nécessité vitale pour la colonie, mais cette occupation n'illustre qu'une partie de l'acti-

$37 \mathrm{Ce}$ nombre comprend 85 personnes qui ont obtenu une concession en 1667 et 30 autres qui ont reçu la leur en 1668 par un acte passé devant le notaire Paul Vachon.

38 Pierre-Georges Roy, Ordonnances, commissions ... op. cit., 63.

39 Talon à Colbert, 13 novembre 1666, RAPQ (1930-31): 58. 
vité des Québécois. Et si l'on pratiquait une agriculture de subsistance, le reste des produits de la terre constituait tout de même un aspect important de l'activité de l'habitant canadien, au milieu du XVII ${ }^{\mathrm{e}}$ siècle.

\section{Le commerce}

L'agriculteur québécois partageait donc son temps entre la culture de la terre et le commerce de ses produits. Disposant de revenus, par la vente de sa surproduction, il devenait acheteur de marchandises utiles ou somptuaires et de services. La présence de 20 marchands ou bourgeois à Québec, à cette époque, indique cette tendance. Les 500 transactions d'affaires qui font l'objet de procès en regard des 35 litiges qui ont rapport à l'occupation de la terre prouvent bien mieux l'importance de l'activité commerciale à Québec au milieu du XVII siècle.

D'ailleurs, l'accession de Richelieu au pouvoir en France avait fait entrer les théories de politique économique dans l'administration gouvernementale. Or, ces théories visaient en définitive le développement du commerce. Faudrait-il croire qu'après 40 ans, en travaillant dans du neuf, la politique cononiale française ait connu un échec complet ? Or, d'une part, le Canada était suffisamment pourvu en capital humain et en ressources, sinon pour jouer un rôle dans le plan de la France, du moins pour que le programme français influençât le développement du Canada. D'autre part, les perspectives de profits, la centralisation du pouvoir et les revenus des habitants et des hommes de métier facilitaient l'extension de ce secteur d'activité. Enfin, on ne peut concevoir la théorie du commerce principal - celui du castor en Nouvelle-France - et les besoins de la colonie, sans tenir compte de ses effets multiplicateurs. Et si le commerce du castor constituait le pôle de croissance de la colonie, l'échange et la vente de produits qui se greffaient à ce secteur primordial pouvaient occuper plus de gens que la traite des pelleteries elle-même.

Les sources consultées fourmillent, par ailleurs, de renseignements intéressants sur l'économie québécoise du milieu du $\mathrm{XVII}^{\mathrm{e}}$ siècle. Que de problèmes le paternalisme pouvait engendrer ! Ainsi, Marguerite Leboeuf obtint un délai de trois ans pour rembourser ses créanciers parce que son mari avait été pris par les Anglais sur les côtes françaises. ${ }^{40}$ Un peu plus tard, Jean Frouin, aussi poursuivi pour dettes, prouve que Marguerite Leboeuf lui était redevable de $450 \#$. Comme elle jouissait d'un délai,

40 Jugements et délibérations du Conseil souverain de la NouvelleFrance, I: 390 . 
le Conseil souverain, dans un jugement à la Salomon, ordonna "qu'il sera pourvue au dict Frouin selon l'exigence des cas". ${ }^{41}$ Certains individus fort désireux de s'enrichir, se servaient de moyens condamnables pour atteindre leur but. Ce fut le cas pour l'agent de la compagnie qui survendait des produits afin de réaliser un profit indirect en particulier aux dépens de son employeur. ${ }^{42}$ Un cabaretier encore plus filou tenait deux livres de comptes. Comme le livre du marchand faisait foi de reçu, il arrivait que l'homme d'affaires oubliât d'inscrire certains paiements et traduisait ses clients en cour. ${ }^{43}$ Ces sources rendent possible enfin une étude précise du coût de la vie en NouvelleFrance. Dans le seul registre de la prévôté de Québec en 1667, il a été possible de relever 150 données sur la valeur de divers produits. Et des données si précises qu'elles permettent d'établir une courbe mensuelle des prix du blé.

\section{Le travail}

Dernier aspect de l'activité des Québécois, mais non le moindre, le travail des hommes de métier. La diversité des besoins requis pour bâtir un pays rendait nécessaire le recours à un grand nombre d'hommes exerçant les métiers les plus divers, offrant leurs services et mettant leur compétence à la disposition de la population québécoise. L'importance numérique des hommes de métier qui figurent au recensement de 1666, la diversité de leur occupation et les nombreux procès faisant suite à la location de services humains démontrent que l'immigration en Nouvelle-France correspondait généralement aux besoins de la colonie. Ajoutons, sans plus insister, que ces documents caractérisent, en outre, le mode de vie d'une partie importante de la population.

\section{La justice}

En 1667, Talon décrivait ainsi le peuple: “quoyque composé d'habitans de différentes provinces de France dont les humeurs ne symbolisent pas toujours, il m'a paru assez uny dans tout le temps de mon séjour". 44 C'est pourtant ce peuple uni qui faisait deux fois plus de procès que d'actes notariés en 1667, sans compter que pour un procès-verbal inscrit au registre de la cour, il fallait au moins six étapes de procédure: la requête, l'acceptation de cette requête, la fixation de la date d'audition de la cause,

41 Ibid.: 419.

42 Registres de la Prévôté de Québec, $1667,1^{\mathrm{e}}$ partie, f.34v.

43 Ibid., 16 (1680), f.200v-202.

44 "Mémoire de Talon sur l'état présent du Canada (1667)", RAPQ $(1930-31): 65$. 
la rédaction et la "délivrance" des exploits ou assignations et le procès lui-même. Il y avait là de quoi occuper bien des gens et gagner honnêtement sa vie. L'intérêt de ces registres n'en est que plus grand, puisqu'il traduit plutôt un type de société qu'une "mentalité chicanière". 45

Si les hommes d'âge mur, ayant des responsabilités comparurent le plus souvent en cour, on y dénombre aussi près de 200 femmes, mais moins de $50 \%$ des domestiques qui figurent au recensement de 1666. Certains allèrent en cour assez souvent: 109 , plus de dix fois en deux ans, dont 19 plus de 30 fois. ${ }^{46}$ Les 109 individus qui se présentèrent en cour plus de dix fois représentent bien les principaux secteurs d'activités de la vie à Québec. On y trouve:

30 hommes de métier

22 marchands

21 habitants

13 huissiers et notaires

3 engagés

21 dont l'occupation est inconnue.

Les quatre premiers groupes sont d'importance égale, car les notaires et les huissiers, se présentèrent en cour plus souvent que les autres, agissant souvent comme procurateurs. ${ }^{47}$

\section{Fonctionnement et réalisme de la justice}

$\mathrm{Si}$ la fréquentation assidue des cours de justice par les Canadiens peut illustrer son mode de vie, elle peut aussi refléter la mentalité de la population. Car l'adaptation à la réalité canadienne du système judiciaire français ne se fit jamais de façon improvisée. L'on retrouve dans les cours de justice canadienne

45 Voir la note 23.

46 Le record pour le nombre de comparutions appartient à Romain Becquet avec 88 en deux ans. L'huissier Jean Levasseur le suit de près avec 79. Ces deux personnes toutefois agissent souvent comme procurateurs pour d'autres Québécois. Le record pour un individu n'agissant que pour lui appartient à Vincent Regnault, officiellement maître tonnelier et habitant, mais surtout cabaretier et trafiquant de fourrures. A lui seul, Regnault comparut 78 fois. De plus, sa femme, aux mœurs un peu légères, mais de caractère volontaire, fut appelée 29 fois en cour.

47 Il faut prendre garde de ne pas confondre procurateur et procureur. Ce dernier représente les intérêts supérieurs: roi ou seigneur; c'est un officier de justice. Le procurateur est une personne engagée par l'une des parties pour agir comme son représentant devant la cour. Certains procurateurs étaient si occupés qu'ils ont dû tirer de ce travail des revenus fort intéressants. 
la même terminologie précise et détaillée qu'en France. Ainsi, le conseil souverain décrète des arrêts et le juge de la Prévôté, des sentences. $^{48}$ A la Prévôté de Québec, on faisait des requêtes verbales, civiles, personnelles ou hypothécaires; les jugements de cette cour étaient définitifs, préparatoires, provisionnels ou interlocutoires. ${ }^{49}$ De plus, les nombreuses étapes à franchir avant de se présenter en cour ne semblent avoir ennuyé personne, pas même les illettrés.

Talon voulait procurer à la colonie une justice gratuite et rapide..$^{50}$ Il rédigea à cette fin un projet de règlement pour alléger le fonctionnement de l'appareil judiciaire canadien. ${ }^{51}$ Toutes les requêtes en première instance ou en appel devaient lui être adressées, et c'est lui qui référait la cause à la cour de son choix. ${ }^{52}$ Une requête pouvait être verbale ${ }^{53}$ et Talon recommandait même que leur présentation se fasse le dimanche pour épargner du temps. ${ }^{54}$ On vit même la Prévôté rendre un jugement le dimanche pour la commodité des parties. $^{55}$ La requête acceptée, la partie demanderesse ou l'office du juge - en l'occurrence le huissier - avertissait la partie poursuivie de se présenter en cour. ${ }^{56}$ Le demandeur devait toutefois faire part des chefs d'accusation qu'il entendait porter pour permettre au défendeur de préparer sa réponse. Un avis donné par le poursuivant en présence d'un voisin digne de foi avait force d'assignation. Avant de comparaître toutefois, les parties devaient tenter de s'entendre à l'amiable en faisant appel à des arbitres. ${ }^{57}$ Quand un litige était soumis directement au Conseil souverain, c'est habituellement la cour de justice elle-même qui désignait les arbitres. On ne connaît pas de façon précise l'efficacité de ce recours à des arbitres. Toutefois, sur 192 cas de défauts - défauts de comparaître - devant la Prévôté de Québec en 1667, 104 litiges n'eurent

${ }^{48}$ L'on devine facilement combien il serait intéressant, à cette fin, de pouvoir établir des comparaisons avec les bailliages ou les juridictions royales des provinces françaises de la côte atlantique.

49 Selon Trévoux, un arrêt est un jugement souverain et en dernier ressort; une sentence est le jugement d'un juge.

50 Lucile Labrèque, Inventaire analytique de la Prévôté de Québec, tome II. Manuscrit non publié conservé aux Archives du Québec.

51 P.-G. Roy, Ordonnances ... op. cit., 51-54.

52 Jugements et délibérations. . .op. cit., 447-448.

53 Jugements et délibérations... op. cit., 447-448. Courcelles refusa de signer cette ordonnance qui, disait-il, lui enlevait des pouvoirs. Talon eut comme principe de reléguer à la Prévôté toutes les réclamations de moins de 10 livres.

54 Ibid., 465.

55 P.-G. Roy, Ordonnances. .. op. cit., 51-54.

56 ł egistres de la Prévôté de Québec, $1667,2^{\mathrm{e}}$ partie, f.50v.

57 Ibid., note 51 . 
aucune suite en cour. Mais, d'une part, il peut y avoir eu plus de requêtes que de défauts; d'autre part, deux facteurs peuvent expliquer cette absence de répercussion en cour: l'entente à l'amiable ou la crainte des frais de cour et de la sentence.

Le déroulement d'un procès se faisait avec minutie: il $\mathrm{y}$ avait revision de toutes les pièces, appels occasionnels à des témoins ou à des experts, exposé des prétentions de chacune des parties, opinion du procureur et enfin la sentence.

C'est à ce dernier niveau, celui des sentences, que se précise le mieux le caractère de l'administration de la justice en NouvelleFrance. En dépit de certaines irrégularités occasionnelles, une hiérarchie de sentences permettait à la cour d'assurer le bon fonctionnement de l'appareil judiciaire. Ainsi l'imposition des dépens - frais de cour - manifeste le réalisme et l'importance accordée au simple bon sens dans le domaine judiciaire. Ces frais de cour étaient toujours à la charge du perdant (192 cas devant la Prévôté en 1667); par contre une cause difficile, où les parties étaient de bonne volonté, n'entraînait pas de frais ( 22 cas spécifiques en 1667). Mais si l'une des parties faisait preuve de mauvaise volonté, elle pouvait encourir une amende en sus des dépens. Quand les deux parties refusaient de collaborer, le juge les mettait hors de cour (39 cas devant la Prévôté en 1667) empêchant ainsi toute possibilité de recours sur le même sujet. Enfin, l'individu qui dépassait les bornes de la raison et du bon entendement pouvait être condamné pour "fol appel", ce qui entraînait une amende automatique.

L'analyse et la comparaison des documents de justice révèlent bien d'autres aspects intéressants. Ainsi, la cour d'appel i.e. le Conseil souverain - réduisait presque toujours la sentence donnée par le juge de la Prévôté. Logiquement, la répétition de ces changements aurait dû inciter le juge de la Cour inférieure à se mettre au pas. Mais il ne l'a pas fait. Son prestige et sa compétence n'étaient donc pas en jeu. Doit-on y voir une coutume à tel point acceptée et passée dans les mœurs que la justice se faisait exemplaire aux niveaux inférieurs et paternaliste aux niveaux supérieurs?

Par ailleurs, l'imposition des frais de cour, qui se percevaient à tous les niveaux et qui comprenaient le paiement des "vacations" des juges, des assignations faites par les huissiers ainsi que le travail des greffiers détruit bien des avancés concernant la gratuité de ce service et les salaires des officiers de justice. D'une part, à cause des frais élevés, le Québécois ne pouvait se payer le luxe ou le plaisir d'intenter des procès sans raison vala- 
ble; et alors, il est faux d'affirmer que le Canadien se plaisait à faire des procès pour des "bouts de terre": ce n'est certes pas le cas à Québec au milieu du XVII ${ }^{\mathrm{e}}$ siècle. Il est, par contre, juste de voir dans cette abondance de procès, une illustration du type ou du caractère de la société de l'époque plutôt que d'une mentalité. D'autre part, l'analyse de la répartition de ces frais de cour prouve que les officiers de justice gagnaient un salaire bien plus élevé que celui fixé par les ordonnances. Des 192 impositions de dépens par la Prévôté de Québec en 1667, 39 précisent le montant à payer qui atteint $166 \#, 5$ sols. ${ }^{58}$ Le calcul de la moyenne et l'extension de cette moyenne à tous les cas dûment certifiés établit à $800 \#$ les revenus à partager entre les officiers de cette cour. Cette donnée, pour une part, contribue à remettre en cause l'approche traditionnelle de cette catégorie de gens puisqu'ils faisaient un revenu supérieur à ce qu'on a dit. Elle fausse aussi bien des comparaisons de traitements faites à partir du salaire théorique de ces officiers. D'un autre côté, il faut réaliser que l'historiographie traditionnelle a généralement considéré les fonctions judiciaires comme un mode de vie à temps plein alors que les officiers de justice n'exerçaient cette fonction que deux jours par semaine et que bien loin de rechercher en dehors de leurs fonctions un revenu suffisant pour vivre, ils ont utilisé le prestige ou les revenus de leur emploi régulier pour accaparer un poste dans la justice. ${ }^{59}$

\section{L'ADMINISTRATION}

Ajoutons à cela que la tâche des officiers de justice était beaucoup plus importante que le simple règlement des litiges. En effet, au début du règne absolutiste et centralisateur de Louis XIV, le gouvernement royal de la Nouvelle-France n'avait pas atteint sa forme parfaite: l'administration du Canada comportait encore de sérieuses dérogations à la hiérarchie des fonctions. Comme en France d'ailleurs, certaines administrations conservaient et exerçaient des pouvoirs non dûment sanctionnés par les édits du roi..$^{60}$ Cette décentralisation du pouvoir se manifeste bien, par exemple, au Conseil souverain qui pouvait com-

58 P.-G. Roy, Ordonnances. .. op. cit., 51-54.

59 A noter qu'il ne fut pas tenu compte de l'un d'eux qui s'élevait à 129 livres parce que le paiement en fut suspendu par suite de l'appel au Conseil souverain, mais surtout pour éviter toute possibilité de surestimer ces frais.

60 C'est le cas, entre autres, du marchand Jacques Le Ber, riche de plus de 200,000 livres et qui occupe un office au bailliage de Montréal. Est-ce là l'exemple d'un bourgeois qui essaie de tout contrôler et d'orienter l'administration à son profit ? 
mettre des gens pour faire enquête ou régler des litiges. ${ }^{61}$ Bien plus, le "gouvernement" d'un pays se fait normalement par voie de réglementation, de lois, ou d'ordonnances; or, à Québec, en 1667 et en 1668, cette réglementation provenait de trois niveaux administratifs différents: du gouverneur ou de l'intendant, du Conseil souverain et de la Prévôté de Québec. L'étude du contenu de ces ordonnances n'a toutefois de valeur pour une si courte période que si elle permet de préciser la portée des unes par rapport aux autres, afin de déterminer s'il y a partage de juridiction au niveau administratif entre chacune des administrations concernées. Et cela est possible même si l'on ne sait pas comment étaient diffusées, appliquées et respectées les ordonnances issues des niveaux inférieurs. En fait, aux trois niveaux, l'on trouve des ordonnances à portée limitée à un individu et d'autres étendues à toute la population. ${ }^{62}$ De plus, même si à une occasion le Conseil souverain reprit une ordonnance émise d'abord par la Prévôté, il ne semble y avoir eu aucune tentative de la part des autorités supérieures pour réprouver ou approuver les ordonnances émises à un niveau inférieur.

Ces règlements enfin se rapportaient toujours à un problème concret, qu'il ait été général ou particulier. Ce réalisme constitue donc une caractéristique essentielle du gouvernement royal de la Nouvelle-France, tout aussi importante que ses aspects absolutiste et paternaliste. Conditionnée, d'une part, par la géographie de la colonie, la mobilité et la diversité d'origine de la population, la politique administrative canadienne ne pouvait être que profondément réaliste.

Cet apercu de la vie à Québec au milieu du XVII ${ }^{e}$ siècle reste bien mince. Il soulève peut-être plus de questions qu'il n'apporte

61 Mandrou, op. cit., 207.

62 Naturellement, les ordonnances de la Prévôté de Québec ne couvraient que la région de Québec; par contre, la juridiction royale de Trois-Rivières et le bailliage de Montréal émettaient aussi des ordonnances.

Par ailleurs, Tracy, Courcelles et Talon firent une ordonnance pour obliger un individu à repasser en France (Roy, Ordonnances ... op. cit., 74), une autre pour procéder à une enquête contre un individu accusé de vol (ibid., 68-69) et une troisième pour permettre aux Jésuites de nommer un passeur sur la rivière Saint-Charles (ibid., 70). Le Conseil souverain, pour sa part, fixa par ordonnances la valeur de la monnaie (Jugements et délibérations... op. cit., 370), le prix de la boisson (ibid., 402 et 468) et le tracé de chemins (ibid., 400). Parmi les 24 ordonnances et avis de la Prévôté, on trouve l'ordre aux volontaires de prendre une concession ou un mâ̂tre (Registres de la Prévôté de Québec, 1667, 2e partie, f.93), la fixation du prix des souliers (ibid., f.36v.), la réparation de chemins (ibid., 76v.) et plusieurs avis particuliers. 
de solutions. Mais il est à remarquer que cette étude a son point de départ dans l'analyse externe des sources plutôt que dans les renseignements que recèlent les documents. En effet, hormis une certaine quantification, il n'est pas tenu compte du contenu de ces sources. Et pourtant, on peut y puiser des précisions susceptibles d'éclairer bien des aspects de notre passé. A l'utilisation et au dépouillement systématique de ces fonds d'archives, jamais encore utilisés, est lié l'avenir de l'histoire de la NouvelleFrance.

JACQUES MATHLeU

Archives du Québec 